\title{
High Precision Spectrum Sensing for Cognitive Radio using Hidden Markov Model
}

\author{
$R$ Vadivelu \\ Assistant Professor, \\ Department of Electronics and \\ Communication Engineering, \\ Sri Krishna College of \\ Technology, Coimbatore \\ 641042 , India
}

\author{
K. Sankaranarayanan \\ Dean, Easa College of \\ Engineering and Technology \\ Coimbatore 641105, India
}

\author{
T. Aswathy \\ P G Scholar, Department of \\ Electronics and Communication \\ Engineering, \\ Sri Krishna College of \\ Technology, Coimbatore \\ 641042, India
}

\begin{abstract}
Efficiency of the wireless communication depends mainly on how the Radio Frequency (RF) spectrum is allocated to the end users. Inadequacy of the RF spectrum resource transpires due to fixed frequency allocation by the regulatory bodies in each region is one of the major problems in allocating it to specific applications. Moreover the allocated RF spectrum is not fully utilized efficiently. Cognitive Radio (CR) is the promising technology used for the detection of the spectrum holes or white spaces, and to reallocate this idle spectrum to Unlicensed Users or Secondary Users (SU) or CR user without causing harmful interference to Licensed Users or Primary Users (PU). In this paper, we present a novel approach for high precession spectrum sensing for CR using Hidden Markov Model (HMM). Current research assumes the presence of a Markov Chain for sub-band utilization by PU, but this consideration has not yet been validated, here we validate the existence of a Markov Chain for sub-band utilization and formulating the HMM for spectrum sensing by Prediction Accuracy (PA). The throughput and accuracy of the proposed method is substantiated using extensive simulations.
\end{abstract}

\section{Keywords}

Cognitive Radio, Hidden Markov Model (HMM), Spectrum Sensing, Markov chain, Sub-band utilization, Prediction Accuracy.

\section{INTRODUCTION}

The Radio Frequency (RF) spectrum is the natural resource for the current wireless communication system, spectrum requirement increased as an exponential increase in the number of wireless standards every day. Recent research by Federal Communication Committee (FCC) shows that an enormous portion of the spectrum is not being occupied by PUs excessively in the frequency range of $3 \mathrm{GHz}[1]$. These unoccupied frequency bands are called spectrum holes or white spaces [2]. Cognitive Radio (CR) is a promising technology used for the detection of spectrum holes and to allocate them to SUs without causing considerable interference to Pus and the spectral efficiency and channel capacity can be improved by the CR.

To sense the resource availability different techniques are used and the optimal technique is the Matched Filter (MF) proposed by Cabric et.al to maximize the received Signal to Noise Ratio (SNR) [3]. MF Method requires the prior knowledge of the PU signal and the threshold level varies with SNR for different signals and the distinction between signal and the interfering noise signals are more difficult, throughput is also a major issue in the dynamic change of the detection time [4]. Further to minimize the spectrum sensing error (SE) by reducing the threshold. Fixing the threshold level leads to the detection of noise factor is feeble and prior knowledge of the PU that cannot be assured at the real-time environment [5], [6] Aleksandar et al. proposed the interference reduction method, in this method the throughput will degrade if the number SUs are added [7]. In the above methods transition from one band to other is done only after detecting whether the band is idle or busy. This may lead to a time delay. If we can envisage the usage pattern of the PU, this time delay can be avoided.

A single cycle detector matches the ideal spectral correlation function for a single value of the cycle frequency proportional with a measured value. It is computationally similar to the optimum energy detector, except that the ideal Power Spectral Density (PSD) is replaced by the ideal spectral correlation function, and the measured periodogram is replaced by the measured cyclic periodogram. A suboptimal version replaces the ideal spectral correlation function with a rectangular window over a band of frequencies in which the signal's spectral correlation function is expected to reside if present [8]. Hidden Markov Model (HMM) is used to predict the usage behavior of a frequency band based on channel usage patterns [9].

The sub-band utilization by PU at any time can be considered as a state, which can be either idle state or unoccupied or busy state or occupied. Existing research assumes the existence of a Markov Chain (MC) for sub-band utilization by the PUs, but this assumption has not been validated. In this paper, we validate the existence of a MC for the sub-band utilization by PUs. Hidden Markov Models (HMMs) can also be used for radio scene classification, case recognition, and making meaningful predictions based on training the past data available [10].

Initially, the state of the PU is modeled as a uniform random sequence for the sake of framework to start with. The true states of the sub-band are unknown to the CR instead of uniform random sequence a Markov Model is applied. Here the state can be either busy or idle. Viterbi Algorithm (VA) is used to find the hidden states of HMM [11]. Performance of this method can be measured by performing extensive simulations using MATLAB.

The rest of the paper is organized as follows. Section 2 covers various issues in Spectrum Sensing system model. HMM concept is introduced in section 3 and section 4 describes the simulation process for the assumed system model. The simulation results are presented in section 5. Finally, section 6 draws the conclusion 


\section{SYSTEM MODEL}

The proposed Spectrum Sensing system model using HMM is shown in Fig.1. PU status is the observed sequence, which is a binary sequence where binary zero represents the channel is in idle state and one assumes the channel is in busy state. It is the true state of the PU, the PU excitation model perform air interface of CR. Based on the status, it will transmit PU signal or noise signal. If the $\mathrm{PU}$ is $\mathrm{ON}$, it may transmit the carrier signal. Here the carrier signal considered is Binary Phase Shift Keying (BPSK) signal. If PU is OFF, it will transmit the noise signal. Spectrum Sensing [12] is the primary function of CR.

Spectrum Sensing Engine (SSE) is created on Energy Detection technique [13]. Here, the energy in the concerned sub-band is measured and compared against the given threshold level. If the energy is greater than the threshold level, then it is considered that the channel is in busy state, otherwise it is sensed to be in idle state. The output of SSE may be prone to errors under the assumption that PU status will follow a MC. So the idea is to get a modified or an accurate expected output. This is supported by HMM. Finally, the predicted state and SSE output are compared with the original PU status for the accuracy of prediction of the proposed method. FFT Averaging Ratio (FAR) algorithm [14] is used to implement energy detection. In FAR algorithm the decision variable is independent of the noise level.

PU status represents true state of the PU. In real time applications the true state sequence of PU is unobservable. Collecting the real time measurements is complex and time consuming one [15]. CR user senses the spectrum band and the sensed data gives rise to the sensed output sequence $\mathrm{X}$. The readings provided by this sensing mechanism are prone to errors in the form of Probability of miss detection Pm and Probability of false alarms Pf [16].

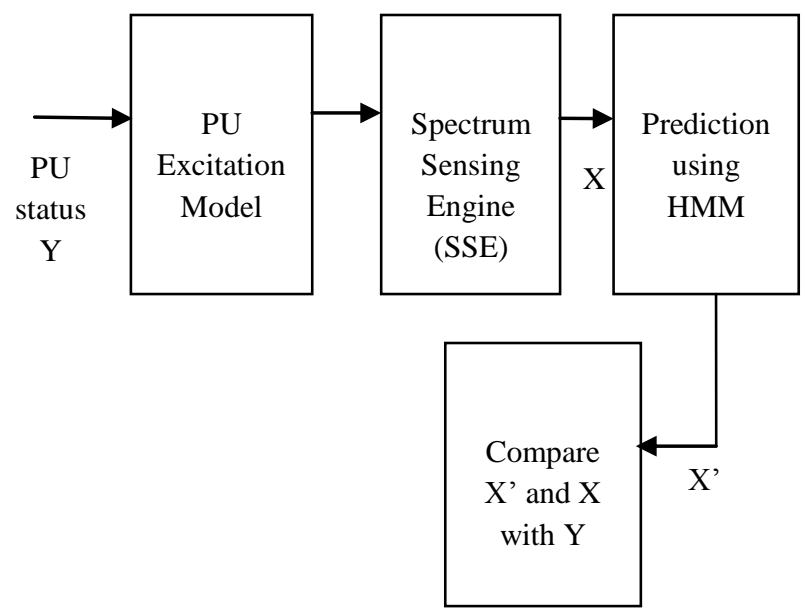

Fig 1: System Model for enhanced Spectrum Sensing

\section{HIDDEN MARKOV MODEL (HMM)}

The main idea of introducing HMM is to model the evolution of occupancy / non-occupancy of a sub-band by its PUs over time using measurements obtained using CR. In this section, we consider the basic operational system model of an HMM.

A HMM is a stochastic process or technique used for the time series of discrete events and this can be continuously distributed either by vectors or scalars. HMM model is doubly embedded stochastic process with an underlying stochastic process that is not observable (it is hidden) and can be only observable through another set of stochastic process that produce the sequence of observation. One of these functions is $\mathrm{MC}$ with finite number of state and the other is a set of random function, referred as alphabet, wherein each function generates a symbol related to a state in the MC. Here the true state $\mathrm{Y}$ of sub-band occupancy is never observable and needed to be sensed using different sensing techniques. Hence, the MC, constituting the true sequence $\mathrm{Y}$, is hidden and the model is characterized as HMM [17], [18].

The general concept of an HMM is illustrated in Fig. 2. Here the states correspond to PU occupancy, i.e., the channel can be either idle or busy with the initial probability , transition probability, emission probability, and the observation, determined using Viterbi Algorithm (VA). Here the transition probability and initial distribution are fixed.

\subsection{HMM and its parameters}

An HMM is characterized by five parameters.

State Space: $\{0,1\}$

Hidden States: $y_{i}$, Observed States: $x$

Transition Matrix: $P=p_{i j}, i=0,1$ and $j=0,1$

Initial Distribution: $p_{0}=\operatorname{Pr}\left(y_{i}=0\right)$ and

$$
p_{1}=\operatorname{Pr}\left(y_{i}=1\right)
$$

Emission probabilities: $e_{y}\left(x_{i}\right)$

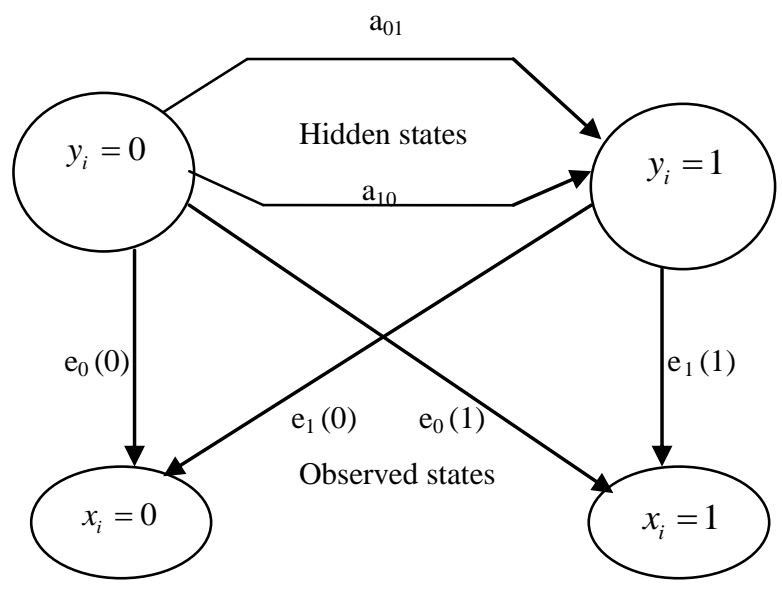

Fig.2 Representation of HMM in spectrum sensing

1) The number of states in the model: - Although, the states are hidden, for real time applications there is often some physical significance related to the states of the model. Generally, the states are interconnected.

2) The number of distinct observation symbols per state: The observation symbols correspond to the physical output of the system being modeled.

3) The state transition probability, $P=\left\{p_{i j}\right\}$ where

$$
\begin{aligned}
P=\left\{p_{i j}\right\} \\
=\operatorname{Pr}\left\langle y_{n}=j \mid y_{n-1}=i\right\rangle---(1)
\end{aligned}
$$

4) The emission probability, $e_{k}(b)$

$$
e_{k}(b)=\operatorname{Pr}\left\langle X_{n}=b \mid Y_{n}=k\right\rangle---(2)
$$


Given appropriate values of above five elements, the HMM can be used as a generator to give an observation sequence.

It can be seen that a complete specification of an HMM requires two model parameters and the specification of three probability measures $P, e_{k}(b)$ and $\pi$ [19].

\subsection{The Viterbi Algorithm}

It is a dynamic programming algorithm used for finding the most likelihood sequence of hidden states in a given sequence of observations. VA can be efficiently computed using a Trellis Structure and the process is as follows

i. Metric Calculation: - It includes path metric and branch metric calculation.

Path metric: - Calculation of the probability of the survivor path.

Branch metric: - Calculation of the probability between input states and the possible output states.

ii. Finding the survivor path: - All other paths are discarded except the maximum path metric.

iii. Trace Back: - It is used to build the correct state by tracing the maximum likelihood path backwards according to the footprints of the previous state.

\section{SIMULATION PROCESS}

We have considered a typical case to validate our proposed model and to determine the prediction accuracy of Viterbi Algorithm (VA). The Transition Matrix is defined as

$$
\text { Transtionmatrix } P=\left(\begin{array}{ll}
0.8 & 0.2 \\
0.9 & 0.1
\end{array}\right)-- \text { (3) }
$$

The initial distribution for each case is determined by the steady-state equation

$$
\begin{gathered}
\left(p_{0} ; p_{1}\right) x P=\left(p_{0} ; p_{1}\right)--(4) \\
p_{0}+p_{1}=1---(5)
\end{gathered}
$$

We have simulated the VA under four different values of probability of errors. In the simulation work for each case, the initial distribution and Transition Matrix $\mathrm{P}$ are fixed. The process consists of the following steps.

- Step 1: Using the initial distribution and Transition Matrix, simulate the Markov chain of length $L=100$ leading to a path $y_{1}, y_{2}, \ldots y_{100}$.

- Step 2: Under each scenario of probability of error, generate $x_{1}, x_{2}, \ldots x_{100}$ using the simulated path $y_{1}^{*}, y_{2}^{*}, \ldots y_{100}^{*}$.

- Step 3: Apply the Viterbi Algorithm to the data $x_{1}, x_{2}, \ldots x_{100}$ to predict the path $y_{1}^{*}, y_{2}^{*}, \ldots y_{100}^{*}$.

- Step 4: Calculate the Prediction Accuracy (PA) by using the formula

$$
P A=\frac{\#\left\{1 \leq I \leq 100: y_{i}^{*}=y_{i}\right\}}{100} x 100
$$

Steps 1 to 4 repeated for 10,000 times. The histogram of these percentages is plotted.

\section{SIMULATION RESULTS}

This section presents the simulated results using MATLAB for the proposed system model. Fig. 3 shows the percentage of accuracy for different number of iterations with the allowed probability of error (PE) of 0.1 and in this case the percentage of the Prediction Accuracy is $95 \%$.

Fig. 4 shows the Prediction Accuracy over the frequency distribution for the probability of error for 0.15 and Fig.5 and Fig.6 shows for the different values of probability of error 0.2 and 0.25. From the Histogram we see that Prediction Accuracy decreases with increase in probability of error and vice versa. For the second case it is about $90 \%$. It is about 85 $\%$ and $80 \%$ for last two cases.



Fig.3 Frequency distribution of Prediction Accuracy percentage for probability of error 0.1

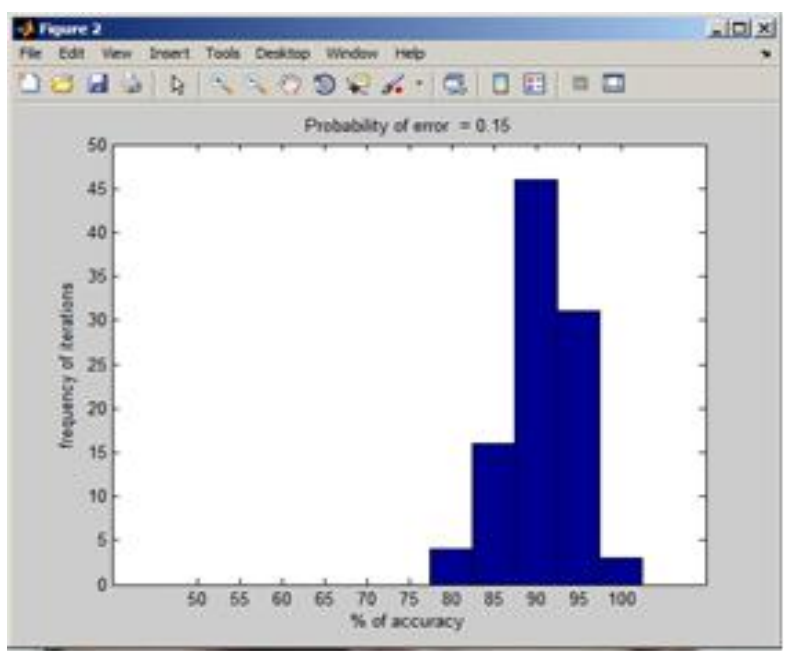

Fig.4 Frequency distribution of prediction accuracy percentage for probability of error 0.15 


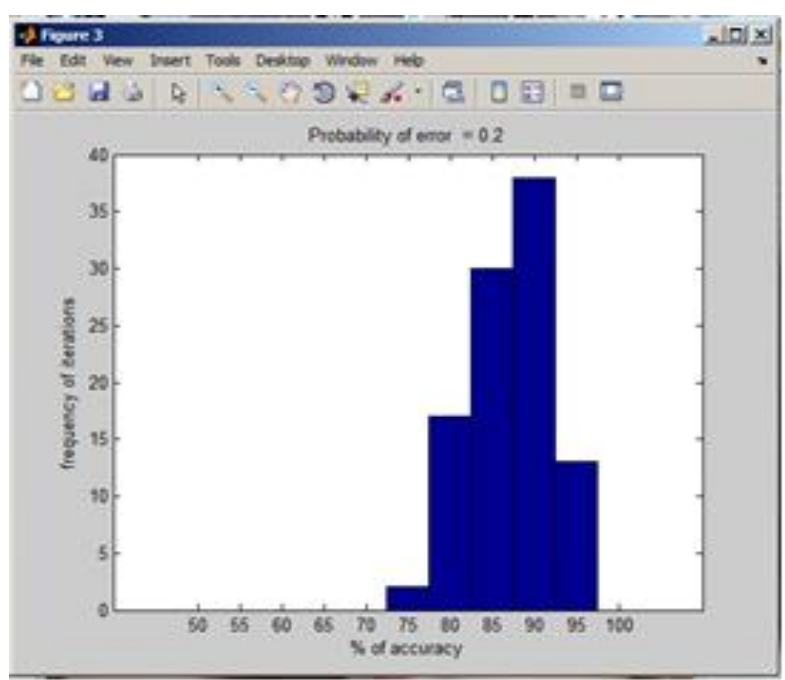

Fig.5 Frequency distribution of Prediction Accuracy percentage for probability of error 0.2 .

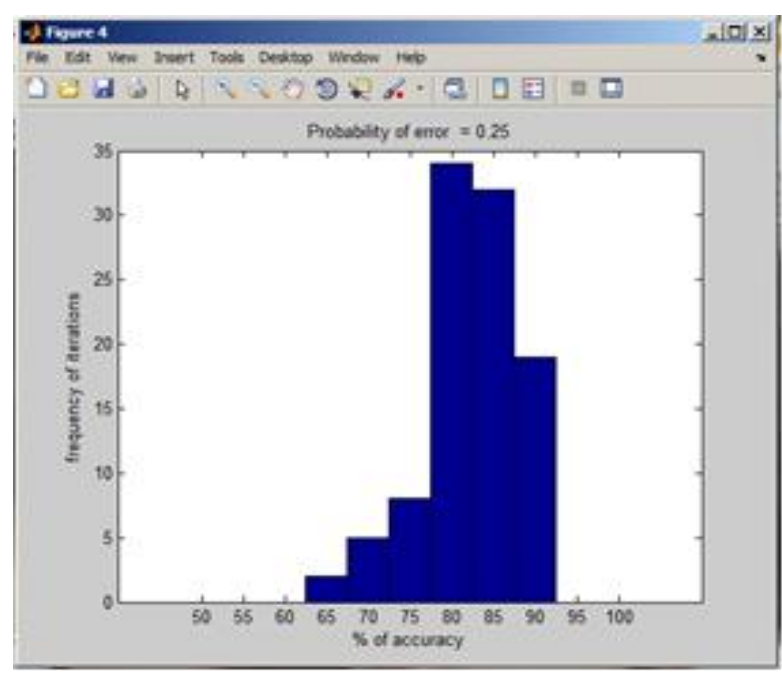

Fig.6 Frequency distribution of prediction accuracy percentage for probability of error $\mathbf{0 . 2 5}$

\section{CONCLUSION}

The requirement of high precision spectrum sensing plays important role in the proper utilization of the spectrum. In real time analysis the true path of the states is hidden to the SU and only the data available to the SU is the sensed data. Hence, the Spectrum Sensing is prone to errors in the form of Miss-Detection (MD) and False Alarm (FA). Therefore, here we exploit these probabilities to frame the spectrum sensing problem into a Hidden Markov Model paradigm. We have used Viterbi Algorithm for implementation of the HMM.

Finally, we have performed MATLAB simulations to illustrate the Prediction Accuracy of the Viterbi Algorithm. We have simulated the Viterbi algorithm for four different values of probability of errors and that shows the percentage of accuracy decreases as probability of error increases.

\section{REFERENCES}

[1] http://www.fcc.gov/oet/info/database/spectrum/

[2] S. Haykin, "Cognitive Radio: Brain-Empowered Wireless Communications", IEEE J. on Selected Areas Commn. Vol 23(2), Feb 2005, pp 221-220.
[ 3 ] Cabric, A., D. Tkachenko, and R. W. Brodersen, Spectrum Sensing Measurements of Pilot, Energy, and Collaborative Detection, Proceedings Military Communications Conference, pp. 1-7, October 2006.

[4] Qun PAN, Xin ZHANG, Ruiming ZHENG, Yongyu CHANG, Dacheng YANG, A Novel Scheme with Adaptive Sampling for Better Spectrum Utilization in Cognitive Radios, Communications and Network, 2009, pp. 46-50.

[5] Dong-Chan Oh and Yong-Hwan Lee, Energy Detection Based Spectrum Sensing for Sensing Error Minimization in Cognitive Radio Networks, International Journal of Communication Networks and Information Security (IJCNIS), Vol. 1, No. 1, April 2009, pp. 1-5.

[6] Kimtho Po., Jun-ichi Takada, 'Signal detection based on cyclic spectrum estimation for cognitive radio in IEEE 802.22 WRAN System ', Technical Report of IEICE.

[7] Aleksandar Jovi cic' and Pramod Viswanath, "Cognitive Radio: An Information-Theoretic Perspective", IEEE TRANSACTIONS ON INFORMATION THEORY, VOL. 55, NO. 9, SEPTEMBER 2009, pp. 3945-3958.

[8] Gardner, W. A., Signal Interception: A Unifying Theoretical Framework for Feature Detection, IEEE Transactions on Communications, 36(8):897-906, 1988.

[9] K.T. Abou-Moustafa, M. Cheriet, C.Y. Suen, "On the structure of hidden Markov models", Pattern Recognition Letters 25 (2004) 923-931.

[10] Schreyogg, C., K. Kittel, and U. Kressel, Robust Classification of Modulation Types Using Spectral Features Applied to HMM, Proceedings of MILCOM, pp. 1377-1381, 1997.

[11] Sung Hwan Sohn, Sung Jeen Jang and Jae Moung Kim, "HMM-based Adaptive Frequency-Hopping Cognitive Radio system to Reduce Interference Time and to Improve Throughput", KSII TRANSACTIONS ON INTERNET AND INFORMATION SYSTEMS VOL. 4, NO. 4, August 2010, pp. 475-490.

[12] Sung Hwan Sohn, Sung Jeen Jang and Jae Moung Kim, HMM-based Adaptive Frequency-Hopping Cognitive Radio System to Reduce Interference Time and to Improve Throughput, KSII TRANSACTIONS ON INTERNET AND INFORMATION SYSTEMS VOL. 4, NO. 4, August 2010, pp. 475-490.

[13] W. A. Gardner, "Signal interception: a unifying theoretical framework for feature detection," IEEE Transactions on Communications, vol. 36, no. 8, pp. 897-906, August 1988.

[14] C. Ghosh, C. Cordeiro, D. Agrawal, and M. Rao, "Markov chain existence and hidden Markov models in spectrum sensing," in Proceedings of IEEE International Conference on Pervasive Computing and Communications (PerCom 2009), 2009, pp. 1 - 6.

[15] Yonghong Zeng, Ying-Chang Liang, Anh Tuan Hoang, and Rui Zhang, "A Review on Spectrum Sensing for Cognitive Radio: Challenges and Solutions", Hindawi Publishing Corporation, EURASIP Journal on Advances in Signal Processing Volume 2010, Article ID 381465, 15 pages. 
[16] Dong-Chan Oh and Yong-Hwan Lee, "Energy Detection Based Spectrum Sensing for Sensing Error Minimization in Cognitive Radio Networks", International Journal of Communication Networks and Information Security (IJCNIS), Vol. 1, No. 1, April 2009.

[17] Z. Chen, N. Guo, and R. C. Qiu, "Demonstration of realtime spectrum sensing for cognitive radio," IEEE Communications Letters, vol. 14, no. 10, pp. 915-917, 2010.
[18] H. Cram'er and M. R. Leadbetter, Stationary and Related Stochastic Processes - Sample Function Properties and Their Applications. New York: J. Wiley \& Sons, 1967. Reprinted by Dover Publ., Mineola, NY, 2004.

[19] T. Koski, Hidden Markov Models for Bioinformatics, Kluwer Academic Publisher, 2001. 